\title{
MAGNETIC FIELDS IN QUASAR 3C147 ON MILLIARCSECOND SCALES
}

\author{
H.Y. ZHANG ${ }^{1,2}$ and R.D. NAN ${ }^{1}$ \\ 'Beijing Astronomical Ohservatory, National Astronomical Observatories, Chinese Academy of \\ Sciences, A20 Datun Road, Chaoyang District, Beijing I000I2, P.R. China \\ ${ }^{2}$ Astronomy Department, Beijing Normal University, Beijing 100875, P.R. China
}

\begin{abstract}
VLBA polarimetric observations of the steep-spectrum quasar 3C147 were made at four frequencies in the available $5 \mathrm{GHz}$ band, from which polarization in one bright feature in the inner jet was detected. The rotation measure of this feature $\left(\sim 1300 \mathrm{rad} \mathrm{m}^{-2}\right)$ agrees with the known high rotation measure of the source, which makes it possible to remove the Faraday screen pixel-bypixel and obtain the intrinsic magnetic field structure. The arch shape of B vectors suggests that a relativistic and distorted jet is being seen in projection.
\end{abstract}

Keywords: VLBI polarimetry, magnetic fields, quasar 3C147

\section{Introduction}

The quasar $3 \mathrm{C} 147(\mathrm{z}=0.545)$ is a steep-spectrum radio source. The VLBI maps of this source by Alef et al. (1988), and Nan et al. (2000) (submitted to A\&A) show complex structure near the core and a well-resolved jet. Using the 45-m telescope at Nobeyama, Inoue et al. (1995) measured a very large integrated rotation measure $(\mathrm{RM})$ for $3 \mathrm{C} 147\left(\mathrm{RM} \sim 1500 \mathrm{rad} \mathrm{m}^{-2}\right)$, suggesting that the radio source may be surrounded by a dense medium.

By observing simultaneously at four different wavelengths in the $5 \mathrm{GHz}$ band with VLBA, Nan et al. (2000) have obtained the distribution of RM across the source, and revealed the structure of the medium causing the Faraday rotation. The authors mapped the intrinsic magnetic fields of 3C147 by removing a simple, uniform RM screen. In this paper, we derive the intrinsic magnetic fields with RM correction pixel-by-pixel, supposing that the mas-scale structure of the screen is real.

\section{Observations and Data Reduction}

The VLBA observations of 3C147 were made in May 1995, at four separate frequenties in the available $5 \mathrm{GHz}$ band, by setting four sets of two BBCs (one for each polarization) to four different wavelengths spread across the band. The data were reduced using the AIPS package and the compact source $0735+178$ was used to 


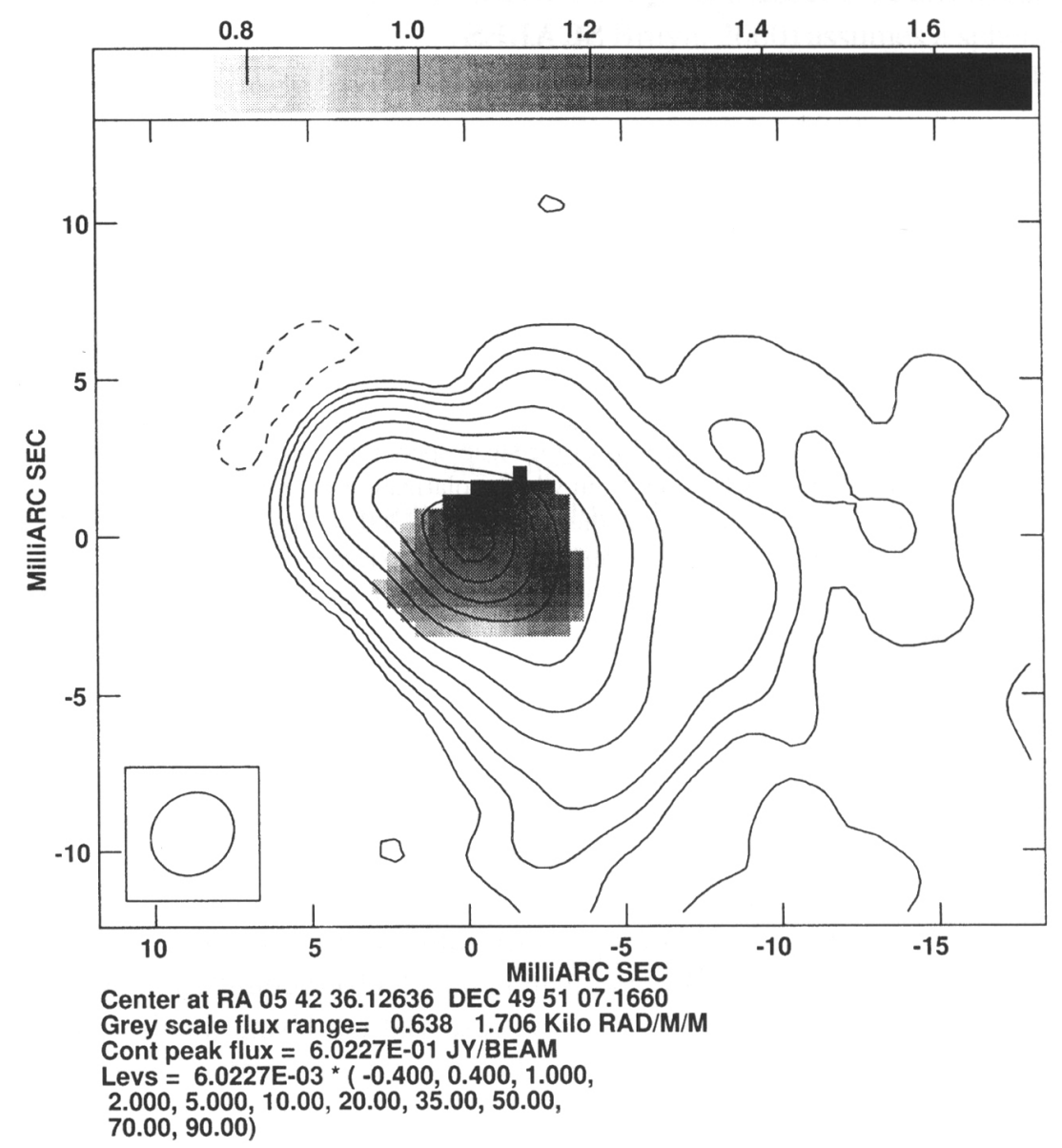

Figure 1. Rotation Measure distribution in the zoomed VLBI total intensity image of the 3C147 core at $4845 \mathrm{MHz}$ where the uncertainty in the local rotation measure is less than $\pm 150 \mathrm{rad} \mathrm{m}^{-2}$. The actual value of RM is the negative of that in this figure.

determined the instrumental polarization parameters ('D-terms') and the absolute polarization position angle $(\chi)$ of $3 \mathrm{C} 147$. Finally the intrinsic magnetic fields on mas stales were determined by using the RM distribution of $3 \mathrm{Cl} 147$ to 'derotate' the observed $\chi$ vectors for the VLBI polarization distribution.

\section{Results and Discussion}

Figure 1 shows rotation measures calculated from the four-frequency data with a blanking error of $\pm 150 \mathrm{rad} \mathrm{m}^{-2}$ as a grey scale map superposed on total-intensity contours. The RM range is from -1706 to $-638 \mathrm{rad} \mathrm{m}^{-2}$. In this Figure, the most 


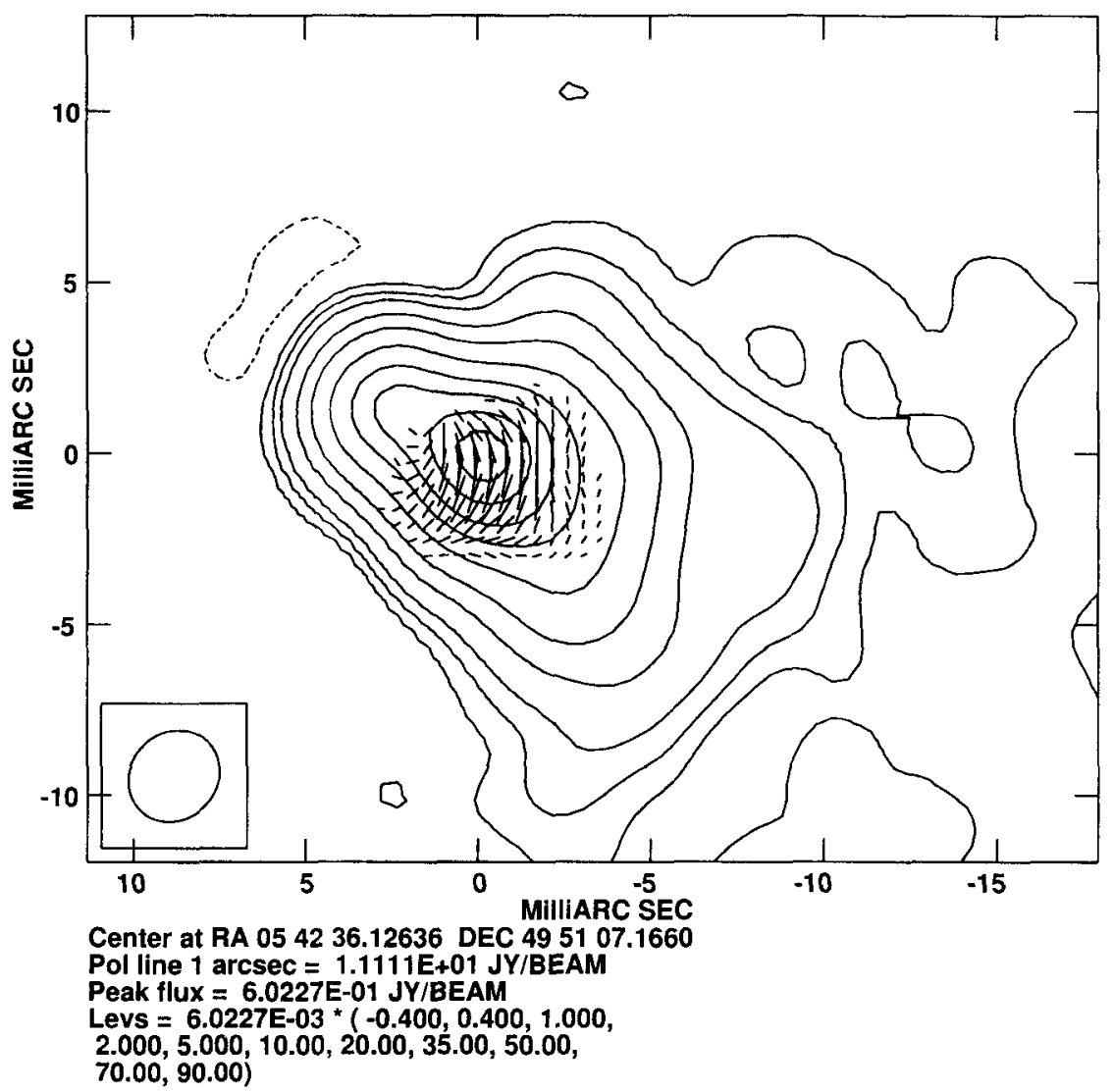

Figure 2. The zoomed VLBI total intensity image of the core $3 \mathrm{Cl} 47$ at $4845 \mathrm{MHz}$ with magnetic ficids vectors superposed. The intrinsic orientation of the $\mathbf{B}$ vectors was determined by 'derotating' the observed $\chi$ vectors using the rotation measure distribution in Figure 1.

reliably observed parts of the RM screen show no connection between the I and RM configuration; this part of the RM distribution is almost uniform, no obvious smooth gradients have been found, which suggests that the thermal plasma giving rise to the large rotation measure of $3 \mathrm{C} 147$ isn't located close to the core emission region. Over the polarized region of $3 \mathrm{C} 147$, the position angle of the plane of polarization is proportional to $\lambda 2$, within the measurement errors, leading to the conclusion that the effect is due to an external screen located near the radio source in a dense sheath around the lobes. From the above discussion, we may propose that the Faraday screen not be closely associated with the inner jet structure, but still located in the source, e.g. a 'cocoon'.

With the high-resolution VLBA observation at $5 \mathrm{GHz}$, the Faraday screen of $3 \mathrm{C} 147$ is resolved on milliarcsecond scales. This enables us to 'derotate' the observed $\chi$ distribution to determine the intrinsic magnetic field direction at all points 
on the mas-scale structure where significant polarization was detected. The inferred projected magnetic fields for $3 \mathrm{C} 147$, corrected for the rotation measure pixel-bypixel, are shown in Figure 2. In the inner part of the B-field map, no large difference has been found from Nan et al. (2000). From Figure 1 and Figure 2, it seems that the B vectors appear somehow aligned with the direction of the weak RM gradients as well, which suggests that the screen may be near the radio source, in a dense sheath around the jet. The arch-like structure of $\mathbf{B}$ fields may imply that the intrinsic $\mathbf{B}$ fields follow a jet, as is common for the inner jets of powerful radio sources, though the jet is not quite visible in our map. The bent vectors might also be caused by very strong projection effects. We have not reached a very satisfactory model to explain what we have mapped, and it is still difficult for us to imagine how the $\mathbf{B}$ fields of this source appear more ordered after they propagate through a non-uniformly distributed medium.

\section{References}

Alef, W., Preuss, E., Kellermann, K.L., Whyborn, N. and Wilkinson, P.N.: 1988, Structural Variability in the Core of 3C147, in: Reid, M.J. and Moran, J.M. (eds.), IAU Symp. 129, The Impact of VLBI in Astrophysics and Geophysics, Kluwer, Dordrecht, pp. 95-96.

Inoue, M., Tabara, H., Kato, T. and Aizu, K.: 1995, Search for High Rotation Measures in Extragalactic Radio Sources I. Multi-Channel Observations at $10 \mathrm{GHz}$, Publ. Astro. Sci. J. 47, $725-737$.

Nan, R.D., Zhang, H.Y., Gabuzda, D.C., Ping, J.S., Schillizzi, R.T., Tian, W.W. and Inoue M.: 2000, High Rotation Measure in the Steep Spectrum Quasar 3C147. Astron. Astrophys, accepted, 2000. 